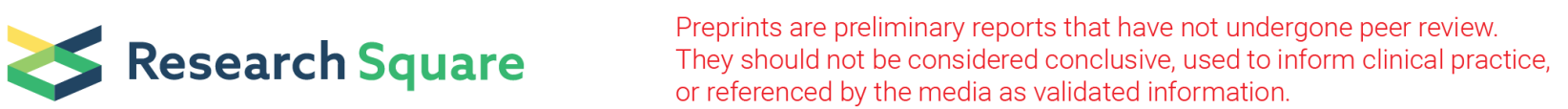

\title{
Clinical Analysis on Diagnostic Accuracy of Bosch Vivalytic SARS- CoV-2 Point-of-Care Test and Evaluation of Cycle Threshold at Admission for COVID-19 Risk Assessment
}

\section{Lukas Andreas Heger ( $\square$ lukas.heger@uniklinik-freiburg.de )}

Department of Cardiology and Angiology I, Heart Center Freiburg University, Faculty of Medicine, University of Freiburg. Freiburg

\section{Nils Elsen}

Department of Cardiology and Medical Intensive Care, Augustinerinnen Hospital, Academic Teaching Hospital University of Cologne. Cologne

\section{Marina Rieder}

Department of Cardiology and Angiology I, Heart Center Freiburg University, Faculty of Medicine, University of Freiburg. Freiburg

\section{Nadine Gauchel}

Department of Cardiology and Angiology I, Heart Center Freiburg University, Faculty of Medicine, University of Freiburg. Freiburg

\section{Urte Sommerwerck}

Department of Pneumology, Augustinerinnen Hospital, Academic Teaching Hospital University of Cologne. Cologne

\section{Christoph Bode}

Department of Cardiology and Angiology I, Heart Center Freiburg University, Faculty of Medicine, University of Freiburg. Freiburg

\section{Daniel Duerschmied}

Department of Cardiology and Angiology I, Heart Center Freiburg University, Faculty of Medicine, University of Freiburg. Freiburg

\section{Marc Oette}

Department of General Medicine, Gastroenterology and Infectious Diseases, Augustinerinnen Hospital, Academic Teaching Hospital University of Cologne. Cologne Ingo Ahrens

Department of Cardiology and Medical Intensive Care, Augustinerinnen Hospital, Academic Teaching Hospital University of Cologne. Cologne 
Keywords: COVID-19, Point of care, PCR test, length of stay, patient flow

Posted Date: October 7th, 2021

DOI: https://doi.org/10.21203/rs.3.rs-944214/v1

License: (c) (1) This work is licensed under a Creative Commons Attribution 4.0 International License. Read Full License 


\section{Abstract}

Background Point-of-care (POC) polymerase chain reaction (PCR) tests have the ability to improve testing efficiency in the Coronavirus disease 2019 (COVID-19) pandemic. However, real-world data on POC tests is scarce.

Objective To evaluate the efficiency of a novel severe acute respiratory syndrome coronavirus 2 (SARSCoV-2) POC test in a clinical setting and examine the prognostic value of on admission cycle threshold (CT) on length of hospital stay (LOS) in COVID-19 patients.

Methods Patients hospitalised between January and May 2021 were included in this prospective cohort study. Patients' nasopharyngeal swabs were tested for SARS-CoV-2 with Allplex ${ }^{\text {TM }} 2019$-nCoV (Seegene Inc.) real-time (RT) PCR assay and novel POC test (Bosch Vivalytic SARS-CoV-2 [Bosch]) as well as the SARS-CoV-2 Rapid Antigen Test (Roche) accordingly. Clinical sensitivity and specificity as well as interand intra-assay variability were analysed.

Results 120 patients met the inclusion criteria with 46 (38\%) having definite COVID-19 diagnose by RTPCR. Bosch Vivalytic SARS-CoV-2 POC had a sensitivity of $88 \%$ and specificity of $96 \%$. The inter- and intra- assay variability was below $15 \%$.

CT Value at baseline was lower in patients with LOS $\geq 10$ days when compared to patients with LOS $<10$ days $(27.82( \pm 4.648)$ vs. $36.2(25.9-39.18) ; p=0.0191)$. There was a negative correlation of CT at admission and LOS $\left(r[44]_{s}=-.31 ; p=0.038\right)$

Conclusion Our data indicate that POC testing with Bosch Vivalytic SARS-CoV-2 is a valid strategy to identify COVID-19 patients and decrease turnaround time to definite COVID-19 diagnosis. Also our data suggests at admission CT as promising marker for length of hospital stay and possibly severity of disease in COVID-19 patients.

\section{Contribution To The Field}

Coronavirus disease 2019 (COVID-19) is a rapidly spreading global pandemic with increased burden on healthcare. Often, centralised laboratory polymerase chain reaction (PCR) testing leads to long severe acute respiratory syndrome coronavirus 2 (SARS-CoV-2) test turnaround times, which in return result in poor patient flow and nosocomial transmission. Point-of-care (POC) PCR tests have the ability to improve testing efficiency by reducing steps between sample collection and results, ultimately contributing to better COVID-19 containment. Numerous assays are suggested for use in clinical practice. However, with some of them just recently released data on their clinical performance is limited. We provide prospective real world data supporting the accuracy and reliability of a new rapid PCR point of care test (Bosch Vivalytic SARS-CoV-2) in a comparative analysis with an antigen test and an extern laboratory. Also our data suggests at admission POC Cycle threshold (CT) as promising marker for length of hospital stay 
and possibly severity of disease in COVID-19 patients. Furthermore our data emphasise the increased risk for long-term health effects of COVID-19 patients and the consecutive need for individual follow-up.

\section{Introduction}

Severe acute respiratory syndrome coronavirus 2 (SARS-CoV-2) with the resulting COVID-19 pandemic has presented hospitals with considerable challenges.(1) These are partly aggravated by long COVID-19 test turnaround times, associated with centralised laboratory PCR testing, resulting in poor patient flow and nosocomial transmission. $(2,3)$ Standard real-time (RT) - polymerase chain reaction (PCR) for COVID-19 is the gold standard but requires specialized materials, equipment, personnel and transportation to a centralized laboratory. (4) Point-of-care (POC) PCR tests have the ability to improve testing efficiency by reducing steps between sample collection and results, ultimately contributing to better COVID-19 containment.(3) Numerous assays are suggested for use in clinical practice. However, with some of them just recently released data on their clinical performance is limited. (5) We compare in a prospective clinical setting Bosch Vivalytic SARS-CoV-2 test for Bosch Vivalytic One with a centralised laboratory PCR test and an established COVID-19 ELISA Elecsys Anti-SARS-CoV-2 assay (Roche).

Current data point towards a plausible positive correlation between the amount of detected virus measured by the proxy PCR cycle threshold (CT) value and the severity of disease. (6) Previous evidence from influenza suggests that the higher the initial viral load, the worse the clinical development. (7) However, firm conclusion on the relationship between initial CT value and individual prognosis is still missing and data for the most part limited to nasopharyngeal viral load samples processed through extern laboratories. (8) Weather CT values derived from POC testing could yield similar results is yet to be established. Different scoring systems have been suggested for COVID-19 risk-assessment, with several of them being derived from established assays. $(9,10)$ Although being influenced by comorbidities and patient characteristics, Length of hospital stay (LOS) has been shown to be a rational choice to measure severity of disease and level of care needed in hospitalized patient. $(11,12)$ Therefore, we strive to evaluate cycle threshold (CT) in POC reverse-PCR tests in confirmed COVID-19 patients as possible marker for LOS and severity of disease.

Such early indicators providing insight into potential disease progression are critical in order to properly select patients requiring special care, especially when exponentially increasing numbers of infections occur in a short time interval and hospitals become overcrowded.

Not only the heterogeneous clinical presentations of COVID-19 poses a challenge for attending physicians but also the prevalence of a variety of late-effects. (13) Divided into two different entities, "post acute COVID-19" where symptoms extend beyond 3 weeks and "chronic COVID-19" where symptoms extend beyond 12 weeks, long COVID-19 is estimated to affect up to $80 \%$. $(14,15)$ However, real-world data for hospitalized patients and long COVID-19 is limited.

\section{Methods}




\subsection{Study design}

We here report data from an investigator-initiated, single-center, prospective registry study to evaluate Vivalytic ${ }^{\text {TM }}$ SARS-CoV-2 Multiplex POC (Bosch) COVID-19 test in clinical practice and to evaluate CT value at admission for risk stratification in hospitalized patients conducted at the Severinenklösterchen Hospital - University of Cologne.

The protocol of this study conforms to the ethical guidelines of the 1975 Declaration of Helsinki and was approved by the institutional ethical committee of the Ärztekammer Nordrhein (20211001).

\subsection{Study population}

All-comers admitted to the hospital over 18 years were eligible for inclusion.

The decision to perform a PCR-test for SARS-CoV-2 was made independently of study inclusion by the treating physician and patients were asked to participate before the test results were available.

Written informed consent was obtained from all patients prior to study inclusion.

Patients with a positive PCR-test for SARS-CoV-2 were finally allocated to the "positive" group, patients with a negative PCR-test for SARS-CoV-2 to the control group (Figure 1). Every patient was then tested 2 additional times using Vivalytic SARS-CoV-2 Test (Bosch), and SARS-CoV-2 Rapid Antigen Test (Rosche) respectively. All nasopharyngeal swabs were taken from nurses or trained personnel.

\subsection{Study plan}

Patient admitted to hospital between January and May 2021 were randomly asked to participate in the study. If patients had agreed to participate, overall characteristics such as sex, age and medical history were recorded.

To characterize the severity of the course of disease in COVID-19 patients were divided according to the length of stay (LOS) in: patients hospitalized for longer or shorter then 10 days .

All clinical data gathered during this period was obtained from the electronic patient file. During follow-up no interventions were applied for the purpose of this study and all therapeutic and diagnostic procedures were applied as part of standard care at the discretion of the treating physicians. Finally, participants were contacted by phone and asked about the course of disease using a standardized questionnaire after 3 month. Herein, the patients were asked to rank their current physical fitness on a scale from $0-100 \%$ in relation to the COVID-19 disease.

\subsection{Measurements}


SARS-CoV-2 testing using Allplex ${ }^{\mathrm{TM}}$ 2019-nCOV Assay (Seegene, Seoul, Korea: polymerase chain reaction assay (PCR) targeting envelope protein- (E-), RNA-dependent RNA polymerase- (RdRP-), and N- genes) was performed by an extern laboratory with Applied Biosystems ${ }^{\text {TM }} 7500$ Real-Time PCR System according to the manufacture guidelines.

For analysis with Bosch Vivalytic SARS-CoV-2 assay (Bosch Healthcare Solutions GmbH, Waiblingen, Germany) using the Vivalytic SARS-CoV-2 analyzer samples were collected in a guanidine thiocyanatebased medium eNAT ${ }^{\circledR}$ (COPAN Diagnostics Inc.; Murrieta, USA), stabilizing the viral RNA and completely inactivating the microbial viability. Vivalytic SARS-CoV-2 analyzer is a portable device and works fully automatically.

Roche SARS-CoV-2 Rapid Antigen Test is an immunochromatographic assay for qualitative detection of SARS-CoV-2 infection in nasopharyngeal swabs. The presence of viral antigens in sufficient concentration enables binding to specific mouse monoclonal anti-SARS-CoV-2 antibodies, which is then reflected by the appearance of a visual indication after clinical specimen is collected and then deposited in a pre-filled extraction buffer container with the solution being put on the test sample according to manufacture guidelines.

Inter-assay variability was performed conducting repetitive tests on four samples throughout three days. Intra-assay variability was performed conducting four consecutive assays in one run. Both times we were using inactivated whole pathogen sample: AMPLIRUN® TOTAL SARS-CoV-2 RNA Control (Vircell SA, Granada, Spain).

\subsection{Statistic}

Continuous patient data were compared using a Mann-Whitney U-test or unpaired T-test. Categorical differences between patient groups were compared using Fishers exact test. Continuous variables are presented as mean \pm standard deviation (SD) if found to follow a Gaussian distribution according to the D'Agosstino-Pearson omnibus normality test or as median \pm lower and upper quartiles if found to follow a non-Gaussian distribution. Categorical patient characteristics are presented as percentages. Spearman nonparametric correlation was used to evaluate the measures the degree to which LOS and CT value at admission move in relation to each other.

Descriptive analyses were performed using Graph Pad Prism Version 9.0 (Prism 9 for Mac OS X; GraphPad Software. Inc.. La Jolla. CA).

\section{Results}

\subsection{Baseline characteristics}


We included 120 patients hospitalized between January and May 2021 in this prospective cohort study. Of those 46 (38\%) patients were tested positive for COVID-19 infection via the extern laboratory. The COVID-19 positive group was younger when compared to the negative control group (55.5 [38.5 - 65.75] years vs. $63.05[ \pm 17.41]$ years; $p=0.007)$.

Patients with COVID-19 disease had higher levels of LDH (362.3 [ \pm 136.1$] \mathrm{U} / \mathrm{I}$ vs. 236 [203.3 - 301]; $\mathrm{p}=0.014)$ and CRP $(5.96[ \pm 5.348] \mathrm{mg} / \mathrm{dl}$ vs. $0.4[0.4$ - 2.7] $\mathrm{mg} / \mathrm{dl} ; \mathrm{p}<0.001)$ when compared to the control group. If the cut-off for a normal value for LDH was considered to be $250 \mathrm{U} / \mathrm{I}$, an increased level of LDH at baseline had a sensitivity of $75 \%$ and a specificity of 53.5\% (PPV 50\%; NPV 77.5\%) for COVID-19 diagnosis. There was no difference with anamnesis for coronary artery disease (8 [17.4\%] patients vs. 15 [20.3\%] patients; $p=0.495)$, hypertension (18 [39.1\%] patients vs. 36 [48.6\%] patients; $p=0.349$ ) or pulmonary disease (9 [19.5\%] patients vs. $25[33.9 \%]$ patients; $p=0.101)$. Symptoms of COVID-19 patients included dyspnea (39.1\%), cough (30.4\%), fatigue (37\%), gastrointestinal complaints (17.4\% [Abdominal pain, diarrhea and anosmia]), fever (41.3) and chest pain (8.7\%). The majority of COVID-19 patients were affected by the virus variant B1.1.7 (31 [67.4\%] of all included COVID-19 patients. (Table 1) 
Table 1

Patients baseline characteristics at admission.

\begin{tabular}{|c|c|c|c|c|c|}
\hline Variable & $\begin{array}{l}\text { COVID-19 } \\
n=46\end{array}$ & & $\begin{array}{l}\text { Non-COVID-19 } \\
n=74\end{array}$ & & $\begin{array}{l}\text { P- } \\
\text { Value }\end{array}$ \\
\hline Age (years) & 55.5 & $\begin{array}{l}38.5- \\
65.75\end{array}$ & 63.05 & \pm 17.41 & 0.006 \\
\hline Sex (female) & 15 & $(33.3 \%)$ & 39 & $(52.7 \%)$ & 0.057 \\
\hline C-reactive protein (mg/dl) & 5.96 & $( \pm 5.348)$ & 0.4 & $(0.4-2.7)$ & $\begin{array}{l}<.001 \\
0.01\end{array}$ \\
\hline Hemoglobine /g/dl) & 13.46 & $( \pm 2.068)$ & 12.79 & $( \pm 2.164)$ & 0.094 \\
\hline Leukocytes (Tsd/ul) & 6.75 & $\begin{array}{l}(4.575- \\
9.85)\end{array}$ & 7.9 & $(6.15-10.2)$ & 0.169 \\
\hline Platlets (Tsd/ul) & 217.5 & $\begin{array}{l}(179- \\
261.8)\end{array}$ & 258.5 & $\begin{array}{l}(195.3- \\
310.3)\end{array}$ & 0.116 \\
\hline $\begin{array}{l}\text { Lactate dehydrogenase } \\
(\mathrm{U} / \mathrm{I})\end{array}$ & 362.3 & $( \pm 136.1)$ & 236 & $203.3-301$ & 0.014 \\
\hline $\begin{array}{l}\text { Aspartate } \\
\text { Aminotransferase }(\mathrm{U} / \mathrm{I})\end{array}$ & 38 & $(26.5-55)$ & 24 & $17-31.25$ & 0.614 \\
\hline$C A D^{*}$ & 8 & $(17.4 \%)$ & 15 & $(20.3 \%)$ & 0.495 \\
\hline Hypertension & 18 & $(39.1 \%)$ & 36 & $(48.6 \%)$ & 0.349 \\
\hline Pulmonary disease & 9 & $(19.5 \%)$ & 25 & $(33.9 \%)$ & 0.101 \\
\hline Length of Stay (days) & 9.5 & \multicolumn{4}{|l|}{$\begin{array}{l}(4.75- \\
15.25)\end{array}$} \\
\hline \multicolumn{6}{|c|}{$\begin{array}{l}{ }^{*} \mathrm{CAD}=\text { Coronary Artery Disease; Continuous variables are presented as mean } \pm \text { standard deviation } \\
(\mathrm{SD}) \text { if found to follow a Gaussian distribution according to the } \mathrm{D} \text { 'Agosstino-Pearson omnibus } \\
\text { normality test. or as median } \pm \text { lower and upper quartiles if found to follow a non-Gaussian } \\
\text { distribution. }\end{array}$} \\
\hline
\end{tabular}

\subsection{Bosch Vivalytic SARS-CoV-2 assay precision}

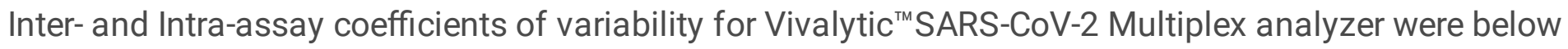
15\%. (Table $2 A$ and $B$ )

In relation to the results of the established extern laboratory PCR test, the POC test had a test-sensitivity for SARS-CoV- 2 infection of $88 \%$ with a specificity of $96 \%$. The positive predictive value was $90 \%$ and the negative predictive value $95 \%$.

The antigen-test had a sensitivity for COVID-19 infection of $65 \%$ with a specificity of $100 \%$. The positive predictive value was $100 \%$ and the negative predictive value $88 \%$. 
Table 2

Inter- (A) and intra- (B) test variability for different test samples L1-L4 and $\mathrm{T} 1-\mathrm{T} 4$

\begin{tabular}{|llllllll|}
\hline A & Ct-Wert & Ct-Wert & Ct-Wert & Mittelwert & SD & VK & VK\% \\
\hline L1 & 31.9 & 31 & 33 & 31.97 & 1.00 & 0.03 & 3.1 \\
\hline L2 & 30.5 & 31.8 & 31.9 & 31.4 & 0.78 & 0.02 & 2.5 \\
\hline L3 & 31.7 & 34.9 & 31.5 & 32.7 & 1.91 & 0.06 & 5.8 \\
\hline L4 & 33.7 & 38.3 & 36.7 & 36.23 & 2.33 & 0.06 & 6.4 \\
\hline
\end{tabular}

\begin{tabular}{|lllllll|}
\hline B & Ct-Wert & Ct-Wert & Mittelwert & SD & VK & Vk\% \\
\hline T1 & 34.1 & 33 & 33.55 & 0.78 & 0.02 & 2.32 \\
\hline T2 & 32.6 & 31.9 & 32.25 & 0.49 & 0.02 & 1.52 \\
\hline T3 & 38.1 & 31.5 & 34.8 & 4.67 & 0.13 & 13.41 \\
\hline T4 & 35.4 & 36.7 & 36.05 & 0.92 & 0.03 & 2.55 \\
\hline
\end{tabular}

\subsection{Length of stay}

Patients with positive SARS-CoV-2 test had an overall length of hospital stay of 9.5 (4.75 - 15.25) days. To quantify severity of disease we divided COVID-19 patients into two groups of patients with LOS $\geq 10 \mathrm{~d}$ (21 [ \pm 17$]$ days) and patients with LOS <10d (4.7 [ \pm 2.7$]$ days).

Patients with LOS $<10 \mathrm{~d}$ had significant lower CT values at admission when compared to patients with LOS $\geq 10 d$ (36.2 [25.9 - 39.18] vs. $27.82[ \pm 4.648] ; p=0.0191)$. (Figure 2 )

Patients with a LOS $<10$ days were younger (45.6 [ \pm 14$]$ years vs. $62.4[ \pm 62.4]$ years; $P=0.001)$ and had higher levels of haemoglobin $(13.6( \pm 1.7) \mathrm{g} / \mathrm{dl}$ vs. $11.9( \pm 2.3) \mathrm{g} / \mathrm{dl} ; \mathrm{p}=0.007)$ when compared to patients with a LOS $>10$ days. There was a negative correlation of CT at admission and LOS $\left(r[44]_{S}=-.31\right.$; $p=0.038$ ). There was no statistical significant difference between both groups regarding other baseline characteristics collected. 15 (75\%) patients in the group with LOS $<10$ days were affected by the virus variant B1.1.7 and $16(61.5 \%)$ patients in the group with LOS $\geq 10$ days $(p=0.4834)$. (Table 3$)$ 
Table 3

Baseline characteristics at admission in patients hospitalized with COVID-19 disease with length of hospital stay (LOS) above or below 10 days respectively.

\begin{tabular}{|c|c|c|c|c|c|}
\hline \multirow{2}{*}{$\begin{array}{l}\text { Variable } \\
\text { LOS* }\end{array}$} & \multicolumn{2}{|c|}{ LOS $<10 d n=23$} & \multicolumn{2}{|c|}{ LOS $\geq 10 d n=23$} & \multirow[t]{2}{*}{ P-Value } \\
\hline & 4.7 & $( \pm 2.7)$ & 21 & $( \pm 17)$ & \\
\hline Age (years) & 45.6 & $( \pm 14)$ & 62.4 & $( \pm 62.4)$ & 0.001 \\
\hline Sex (female) & 7 & $(30.4 \%)$ & 8 & $(34.8 \%)$ & 0.753 \\
\hline C-reactive protein (mg/dl) & 3.6 & $(0.4-7.6)$ & 7.7 & $( \pm 6.3)$ & 0.051 \\
\hline Haemoglobine (g/dl) & 13.6 & $( \pm 1.7)$ & 11.9 & $( \pm 2.3)$ & 0.007 \\
\hline Leukocytes (Tsd/ul) & 7.5 & $( \pm 4)$ & 7.4 & $(4.9-10)$ & 0.628 \\
\hline Platlets (Tsd/ul) & 209 & $(173-257)$ & 233 & $(181-282)$ & 0.652 \\
\hline Lactate dehydrogenase (U/I) & 330 & $(258-469)$ & 377 & $(230-447)$ & 0.989 \\
\hline Aspartate Aminotransferase (U/I) & 38 & $(16-38)$ & 46 & $( \pm 30)$ & 0.941 \\
\hline$C A D * *$ & 2 & $(9 \%)$ & 6 & $(25 \%)$ & 0.119 \\
\hline Hypertension & 7 & $(29 \%)$ & 11 & $(48 \%)$ & 0.567 \\
\hline Pulmonary disease & 4 & $(17 \%)$ & 5 & $(22 \%)$ & 0.710 \\
\hline Virus Variant B1.1.7 & 15 & $(65 \%)$ & 11 & $(48 \%)$ & 0.234 \\
\hline 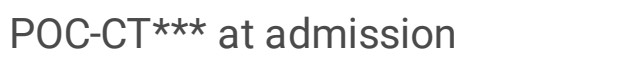 & 36.2 & $(25.9-39.18)$ & 27.82 & $( \pm 4.648)$ & 0.019 \\
\hline \multicolumn{6}{|l|}{ Vital parameters at admission } \\
\hline Mean Pressure (mmHg) & 94.5 & $(81-105)$ & 90 & $(87-94)$ & 0.616 \\
\hline Temperature & 37.6 & $(36.8-38.5)$ & 37.4 & $(36.5-38.1)$ & 0.410 \\
\hline Respiratory Rate (per min.) & 18.3 & $( \pm 4.8)$ & 17.6 & $( \pm 3.2)$ & 0.530 \\
\hline Heart Rate (Beats/minute) & 87.7 & $( \pm 15.1)$ & 92.4 & $( \pm 18.7)$ & 0.367 \\
\hline Saturation at admission (\%) & 96 & $(94.8-98)$ & 95.5 & $(89.5-96.3)$ & 0.127 \\
\hline \multicolumn{6}{|c|}{$\begin{array}{l}\text { * LOS }=\text { Length of stay; } * * C A D \text { C Coronary Artery Disease; } * * * C T=C y c l e \text { Threshold only CT values of the } \\
\text { Point-of Care test (LOS }<10 \mathrm{~d} n=20 \text { and } L O S \geq 10 \mathrm{~d} n=20) \text {; Continuous variables are presented as mean } \\
\pm \text { standard deviation }(S D) \text { if found to follow a Gaussian distribution according to the D'Agosstino- } \\
\text { Pearson omnibus normality test. or as median } \pm \text { lower and upper quartiles if found to follow a non- } \\
\text { Gaussian distribution. }\end{array}$} \\
\hline
\end{tabular}

\subsection{Outcome}

Of 46 patients tested positive, 22 completed the follow-up and 5 COVID-19 patients died. 
At three month after hospital discharge 15 (68\%) COVID-19 patients had persistent depreciation of Quality of life incidental with COVID-19 disease (80 [70 - 90] \% of the normal physical capacity).

\section{Discussion}

In this single centre prospective cohort study we evaluate real-world diagnostic accuracy of point-of-care testing using Bosch Vivalytic SARS-CoV-2 test for Bosch Vivalytic One by comparing sensitivity and specificity to an established extern laboratory as well as a commercially available antigen test.

Our prospective and comparative evaluation shows that Bosch Vivalytic SARS-CoV-2 POC test is a valid strategy for COVID-19 detection with the potential to increase door-to-diagnosis allowing earlier detection of COVID-19 positive patients. $(3,17)$ With its advertised turnaround time of approximately 39 minutes it exceeds those of established extern laboratories using RT-PCT by far. (5) The agreement of the POC test and RT-PCR is good with robust inter- and intra-assay reliably and good detection of positive samples. However, recently published data indicates that in the very early phase of the infection with high CT values, in which the RT-PCR can already detect the infection, the antigen level is not high enough for the POC test, necessitating repeated testing. $(18,19)$

While RT-PCR should still be the gold standard, POC testing could help with patient triage and improve patient flow dynamics. (20) Antigen detection alone proved to be too uncertain for definite COVID-19 diagnosis in line with other publications. (21)

The prognostic value of Cycle Threshold in COVID-19 patients has been suggested by several studies. (22) Prospective data as well as data depending on CT values acquired using POC tests however is missing. Our data shows that on admission POC CT values are lower in patients with prolonged length of hospital stay in COVID-19 patients and that CT value at admission correlates with LOS. Nevertheless, variability of CT values of up to 8 cycles were observed for the same specimen material tested in different laboratory in a recent publication. (23) Therefore, risk assessment using CT values should be only considered if tests are acquired from only one single laboratory using the same assay.

Nevertheless, through their easier implementation, cost effectiveness and accuracy POC COVID-19 test could help monitoring epidemiological features of the COVID-19 pandemic through testing of large cohorts. (24)

Long-term health effects of COVID-19 called Long-COVID or COVID long-haulers are challenging for clinicians through their heterogeneity. (13) It is vital to understand the percentage of patients suffering from long-term effects. Our data shows, that up to $68 \%$ of discharged patients experience a COVID-19 related decrease in quality of life at three month after hospital discharge. This is in line with several other studies and the recommendation for Patients discharged after COVID-19 hospitalization to follow-up with their primary care physician on a regular basis maybe even through telemedicine visits. $(25,26)$ 
Our data depicts the third wave of COVID-19 pandemic in Germany early 2021 with SARS-CoV-2 Variant B.1.1.7 being the predominantly detected variant. (27) This mutant strain prompted border closures, lockdowns and new restrictions ultimately cumulating in further deaths and economic loss. However, with a large proportion of the elderly being already inoculated following the guidelines of the Standing Committee on Vaccination (STIKO) we report COVID-19 patients to be younger then regular hospital allcomers forming our control group. (28) This all-the more emphasises the need for rapid inoculation to protect patients.

Several routine laboratory markers have been suggested in COVID-19 diagnostic algorithm. (29) In a recent meta-analysis Lactate dehydrogenase (LDH) has been suggested as cost-effective biomarker in patients with COVID-19. (30) An important variable in our study is that two distinct patient groups were analysed one composed out of regular all-comers and one of COVID-19 patients. In our prospective data elevated LDH was a distinct feature of COVID-19 patients when compared to the control group. However, LDH was not associated with LOS. Larger clinical trials are needed to confirm the value of LDH at baseline for COVID-19 diagnosis.

\section{Limitations}

POC PCR is a qualitative not quantitative measurement and it is not possible to quantify the virus load via CT values. CT values vary in-between different assays. We used only a single. negative RT-PCR to confirm the absence of COVID-19 infection, risking missing infection. A lot of patients were lost to followup. We did not assess quality of life at the time point of inclusion. The fact, that patients with LOS $>10$ days were younger could possibly diminish the interpretability of our data.

\section{Conclusion}

This data from a single centre provides prospective and comparative data indicating, that POC testing using Bosch Vivalytic SARS-CoV-2 is a valid strategy to identify COVID-19 patients and decrease turnaround time during COVID-19 pandemic. Also our data suggests at admission CT as promising marker for length of hospital stay and possibly severity of disease in COVID-19 patients. Our data emphasise the increased risk for long-term health effects of COVID-19 patients and the consecutive need for individual follow-up.

\section{Declarations}

\section{Ethics approval, accordance and consent to participate:}

The protocol of this study conforms to the ethical guidelines of the 1975 Declaration of Helsinki and was approved by the institutional ethical committee of the Ärztekammer Nordrhein (20211001). Written informed consent was obtained from all patients prior to study inclusion. 


\section{Consent for publication}

Not applicable.

\section{Availability of data and materials}

The datasets analysed during the current study are available from the corresponding author on reasonable request.

\section{Competing interests}

The authors declare that there is no conflict of interest'.

\section{Funding}

This work was not funded.

\section{Authors' contributions}

$\mathrm{LH}, \mathrm{IA}$, and NE designed the study. $\mathrm{LH}$ and NE collected the data. NE and $\mathrm{LH}$ analysed the data. $\mathrm{LH}, \mathrm{NE}$ and IA wrote the manuscript. US, NG, DD, US, CB and MO reviewed and corrected the paper. All authors contributed to the article and approved the submitted version.

\section{Acknowledgments}

DD is a member of SFB1425, funded by the Deutsche Forschungs-gemeinschaft (DFG, German Research Foundation) - Project \#422681845; LAH received funding from the German Research Foundation (DFG; HE 8679/1-1:1)

\section{References}

1. Moynihan R, Sanders S, Michaleff ZA, Scott AM, Clark J, To EJ, et al. Impact of COVID-19 pandemic on utilisation of healthcare services: a systematic review. BMJ Open. 2021;11(3):e045343.

2. Israfil SMH, Sarker MMR, Rashid PT, Talukder AA, Kawsar KA, Khan F, et al. Clinical Characteristics and Diagnostic Challenges of COVID-19: An Update From the Global Perspective. Front Public Health. 2020;8:567395.

3. Brendish NJ, Poole S, Naidu VV, Mansbridge CT, Norton NJ, Wheeler H, et al. Clinical impact of molecular point-of-care testing for suspected COVID-19 in hospital (COV-19POC): a prospective, 
interventional, non-randomised, controlled study. Lancet Respir Med. 2020;8(12):1192-200.

4. Binnicker MJ. Challenges and Controversies to Testing for COVID-19. J Clin Microbiol. 2020;58(11).

5. Kohmer N, Rabenau HF, Hoehl S, Kortenbusch M, Ciesek S, Berger A. Comparative analysis of pointof-care, high-throughput and laboratory-developed SARS-CoV-2 nucleic acid amplification tests (NATs). J Virol Methods. 2021;291:114102.

6. Trunfio M, Venuti F, Alladio F, Longo BM, Burdino E, Cerutti F, et al. Diagnostic SARS-CoV-2 Cycle Threshold Value Predicts Disease Severity, Survival, and Six-Month Sequelae in COVID-19 Symptomatic Patients. Viruses. 2021;13(2).

7. Han A, Czajkowski LM, Donaldson A, Baus HA, Reed SM, Athota RS, et al. A Dose-finding Study of a Wild-type Influenza A(H3N2) Virus in a Healthy Volunteer Human Challenge Model. Clin Infect Dis. 2019;69(12):2082-90.

8. Rao SN, Manissero D, Steele VR, Pareja J. A Systematic Review of the Clinical Utility of Cycle Threshold Values in the Context of COVID-19. Infect Dis Ther. 2020;9(3):573-86.

9. Liu S, Yao N, Qiu Y, He C. Predictive performance of SOFA and qSOFA for in-hospital mortality in severe novel coronavirus disease. Am J Emerg Med. 2020;38(10):2074-80.

10. Satici C, Demirkol MA, Sargin Altunok E, Gursoy B, Alkan M, Kamat S, et al. Performance of pneumonia severity index and CURB-65 in predicting 30-day mortality in patients with COVID-19. Int J Infect Dis. 2020;98:84-9.

11. Rees EM, Nightingale ES, Jafari Y, Waterlow NR, Clifford S, CA BP, et al. COVID-19 length of hospital stay: a systematic review and data synthesis. BMC Med. 2020;18(1):270.

12. Williams GA, Liu J, Chapman WC, Hawkins WG, Fields RC, Sanford DE, et al. Composite Length of Stay, An Outcome Measure of Postoperative and Readmission Length of Stays in Pancreatoduodenectomy. J Gastrointest Surg. 2020;24(9):2062-9.

13. Lopez-Leon S, Wegman-Ostrosky T, Perelman C, Sepulveda R, Rebolledo PA, Cuapio A, et al. More than 50 Long-term effects of COVID-19: a systematic review and meta-analysis. medRxiv. 2021.

14. Carfi A, Bernabei R, Landi F, Gemelli Against C-P-ACSG. Persistent Symptoms in Patients After Acute COVID-19. JAMA. 2020;324(6):603-5.

15. Raveendran AV, Jayadevan R, Sashidharan S. Long COVID: An overview. Diabetes Metab Syndr. 2021;15(3):869-75.

16. Cuschieri S. The CONSORT statement. Saudi J Anaesth. 2019;13(Suppl 1):S27-S30.

17. Dinnes J, Deeks JJ, Adriano A, Berhane S, Davenport C, Dittrich S, et al. Rapid, point-of-care antigen and molecular-based tests for diagnosis of SARS-CoV-2 infection. Cochrane Database Syst Rev. 2020;8:CD013705.

18. Mina MJ, Parker R, Larremore DB. Rethinking Covid-19 Test Sensitivity - A Strategy for Containment. N Engl J Med. 2020;383(22):e120.

19. Stromer A, Rose R, Schafer M, Schon F, Vollersen A, Lorentz T, et al. Performance of a Point-of-Care Test for the Rapid Detection of SARS-CoV-2 Antigen. Microorganisms. 2020;9(1). 
20. Bhandari S, Tak A, Singhal S, Shukla J, Shaktawat AS, Gupta J, et al. Patient Flow Dynamics in Hospital Systems During Times of COVID-19: Cox Proportional Hazard Regression Analysis. Front Public Health. 2020;8:585850.

21. Caruana G, Croxatto A, Kampouri E, Kritikos A, Opota O, Foerster M, et al. Implementing SARS-CoV-2 Rapid Antigen Testing in the Emergency Ward of a Swiss University Hospital: The INCREASE Study. Microorganisms. 2021;9(4).

22. Rajyalakshmi B, Samavedam S, Reddy PR, Aluru N. Prognostic Value of "Cycle Threshold" in Confirmed COVID-19 Patients. Indian J Crit Care Med. 2021;25(3):322-6.

23. Tom MR, Mina MJ. To Interpret the SARS-CoV-2 Test, Consider the Cycle Threshold Value. Clin Infect Dis. 2020;71(16):2252-4.

24. Ade C, Pum J, Abele I, Raggub L, Bockmuhl D, Zollner B. Analysis of cycle threshold values in SARSCoV-2-PCR in a long-term study. J Clin Virol. 2021;138:104791.

25. Understanding the long-term health effects of COVID-19. EClinicalMedicine. 2020;26:100586.

26. Abdelnabi M, Leelaviwat N, Eshak N, Mekraksakit P, Nugent K, Payne JD. COVID-19 discharge and follow-up recommendations. Proc (Bayl Univ Med Cent). 2020;34(1):73-5.

27. Wilton T, Bujaki E, Klapsa D, Majumdar M, Zambon M, Fritzsche M, et al. Rapid Increase of SARSCoV-2 Variant B.1.1.7 Detected in Sewage Samples from England between October 2020 and January 2021. mSystems. 2021;6(3):e0035321.

28. Bogdan C. [STIKO vaccination recommendations : Vaccination of immunodeficient patients and vaccination against COVID-19]. Hautarzt. 2021;72(2):92-9.

29. Stegeman I, Ochodo EA, Guleid F, Holtman GA, Yang B, Davenport C, et al. Routine laboratory testing to determine if a patient has COVID-19. Cochrane Database Syst Rev. 2020;11:CD013787.

30. Martha JW, Wibowo A, Pranata R. Prognostic value of elevated lactate dehydrogenase in patients with COVID-19: a systematic review and meta-analysis. Postgrad Med J. 2021.

\section{Figures}




\section{study population}

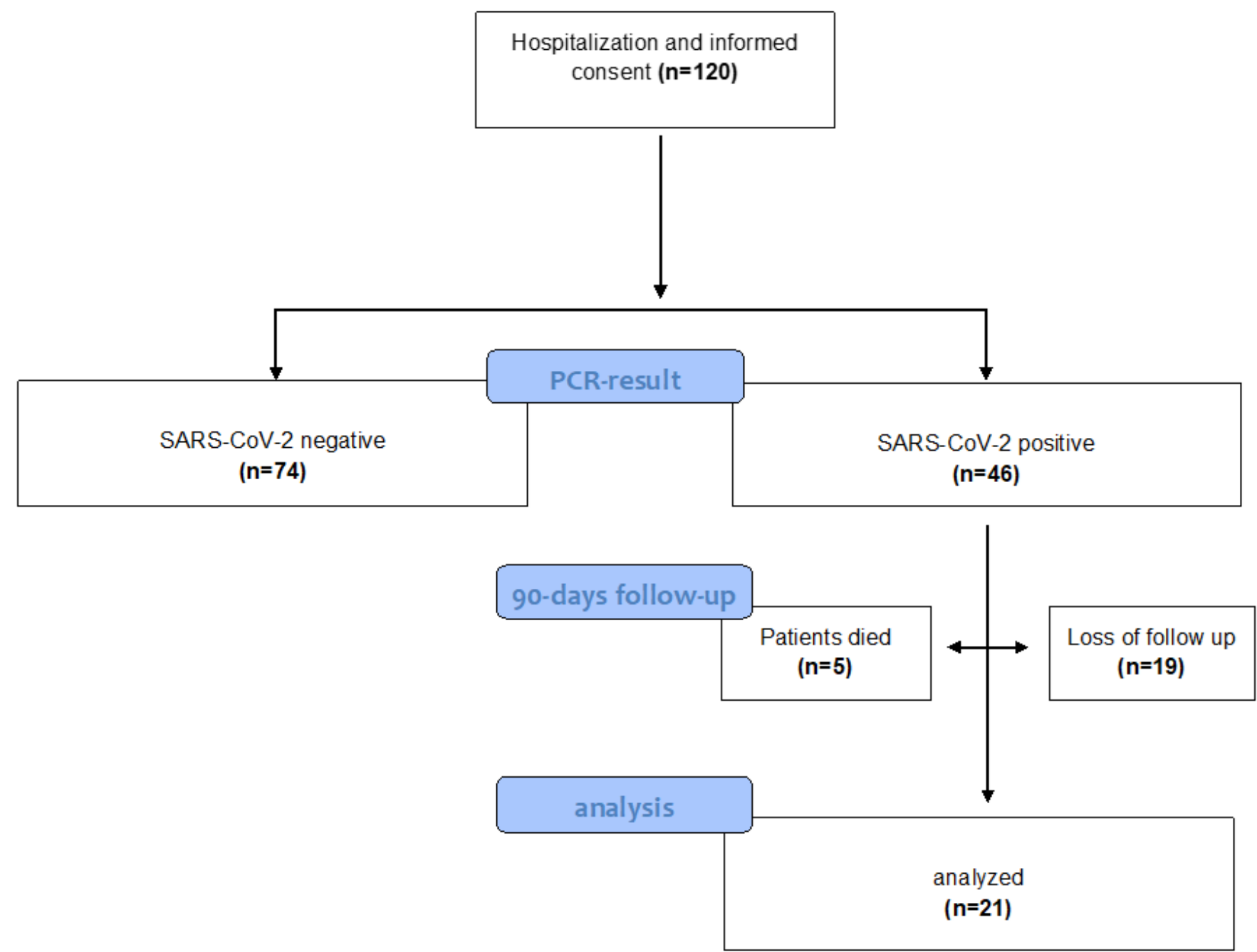

Figure 1

Schematic representation of the allocation to the positive or negative group of the 120 participants. 19 participants of the SARS-CoV-positive cohort were lost to follow-up and 5 participants died. The flow diagram is based on the template of the CONSORT flow diagram. (16) 


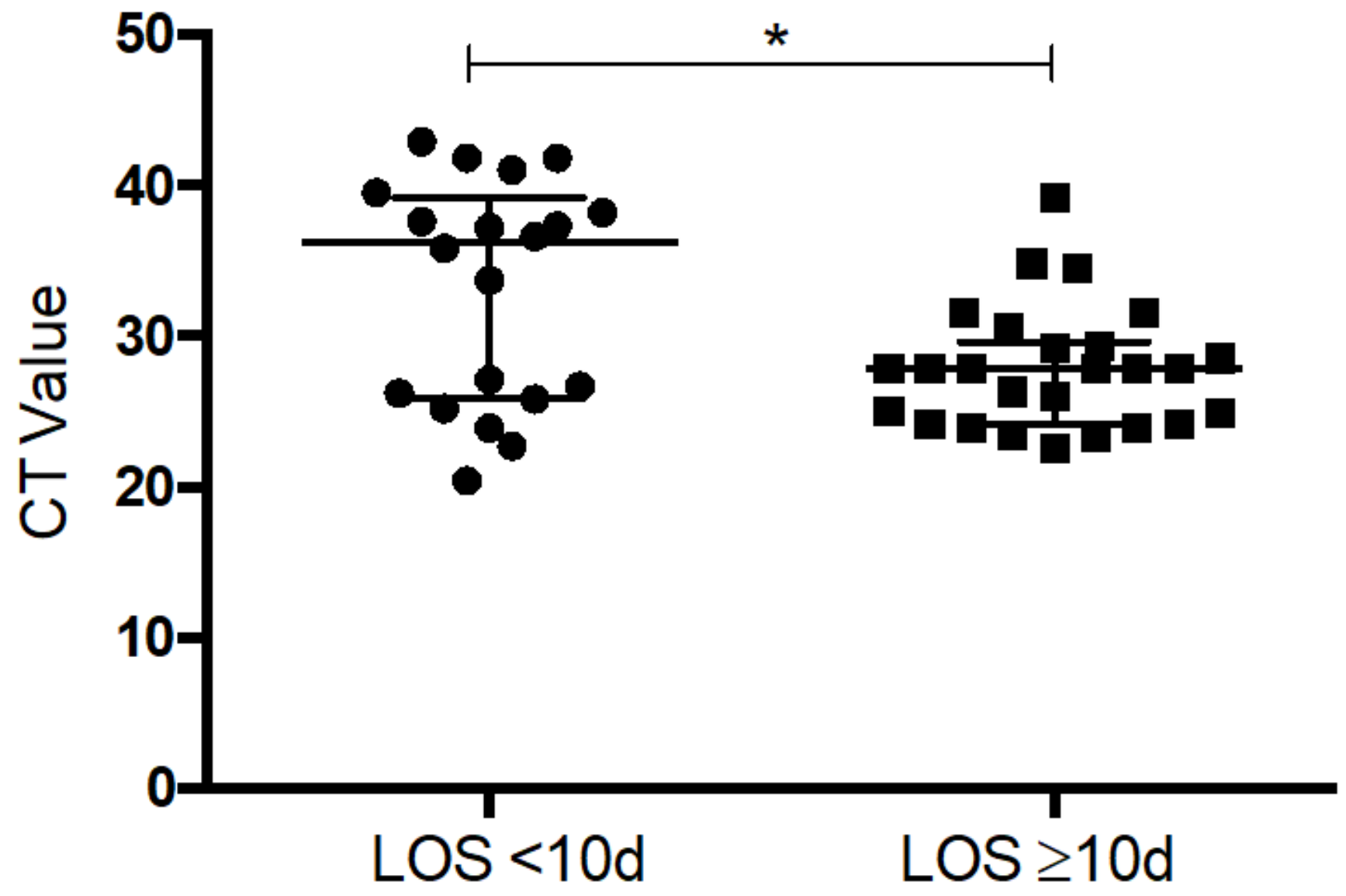

Figure 2

Point-of-Care Cycle- threshold (CT) Value at admission in patients with confirmed diagnose of COVID-19 and length of hospital stay (LOS) above ( $\geq 10$ days) or below (LOS $<10 \mathrm{~d}$ ) ten days respectively. Data are presented as scatter block with Interquartile Range. 